\title{
NUMERICAL SOLUTION WITH GRAPH THEORY FOR FLOOD FLOW IN RIVER NETWORKS
}

\author{
Tuan NGUYEN Le ${ }^{1}$ and Satoru SUGIO ${ }^{2}$ \\ ${ }^{1}$ Student Member of JSCE, Graduate Student, Graduate School of System Engineering, Miyazaki University \\ (Gakuen Kibanadai Nishi 1-1, Miyazaki 889-2192, Japan) \\ 2 Member of JSCE, Dr. of Eng., Professor, Department of Civil and Environmental Engineering, Miyazaki \\ University (Gakuen Kibanadai Nishi 1-1, Miyazaki 889 - 2192, Japan).
}

\begin{abstract}
This paper deals with the flood routing in river networks where the solution can not be obtained from hydrologic routing methods. An improvement for dynamic unsteady one-dimensional (1-D) flow simulation based on Preissmann implicit finite difference scheme with simultaneous solution is developed. The river networks are described by a set of basic river reaches between two nodes. A procedure for automatically creating the order for controlling double-sweep is established. It then is applied for flood routing in the river network of a part of the Red river basin, Vietnam. From the results, the improvement is expected as an effective treatment for dynamic flood routing in river networks of dendritic type.
\end{abstract}

Key Words: numerical solution, graph theory, double sweep method, flood routing, unsteady flow, finite difference method, river networks, Red river basin, Vietnam

\section{INTRODUCTION}

Flood routing is variously defined in hydrology literature. It usually represents a procedure by which to determine the outflow hydrograph at a downstream point of a river (or reservoir), given the inflow hydrograph at an upstream point thereof ${ }^{\text {l) }}$. Flood routing techniques may be classified in two major categories: hydrologic routing and hydraulic routing. The first is simpler than the later. Hydraulic routing is more complex and accurate than hydrologic routing and is based on the solution of continuity equation and momentum equation for unsteady flow in openchannels ${ }^{2}$. The well-known St. Venant equations mathematically describing a gradually varied unsteady flow in open-channels are usually used.

For the subject of flood routing, the finite difference method has been often used. The common numerical methods for implicit finite difference equations are double-sweep method and Newton's iteration method ${ }^{3), 4), 5), 6)}$. In the case of a river network, it needs some modifications as attempts to avoid solving complicated and extremely large coefficient matrices resulting from the full-system approach ${ }^{7)}$. Many researchers have introduced the various techniques for flood routing in the networks as treating flow from a tributary like lateral flow, overlapping segment technique ${ }^{9}$, combining the 1-D and 2-D schemes resulting an inundation model ${ }^{10)}$.

Utilizing graph theory, a network can be described by nodes and links and then the system of equations can be solved by an iterative procedure ${ }^{11), 12)}$ but it is difficult to estimate the time of calculation and the necessary modifications when modeling and simulating a large network ${ }^{13)}$. Graph theory is also used for unsteady flow modeling in river networks with Galerkin method ${ }^{14)}$.

Researches of Choi et al. ${ }^{7)}$ and Ping et al. ${ }^{15)}$ have used the Preissmann scheme with simultaneous solution based on double-sweep method. The research of Choi et al. (and then improved by Nguyen et $a .^{16)}$ ) has limitation on the number of upstream converging or downstream diverging channel segments at each junction ${ }^{7)}$; or personal difficulties from the so-called "general node numbering scheme" and network with junction of more than four branches $^{16)}$. The research of Ping et al. seems to be an alteration of internal boundary processing, not of simultaneous solution ${ }^{15)}$.

In this paper, an improvement of simultaneous solution based on the double-sweep method is introduced and applied for flood routing in river networks. From this improvement, the simultaneous solution can be applied for any branched network regardless of its complexity.

\section{GOVERNING EQUATIONS}

Gradually varied unsteady flows in open-channel are described by the St. Venant ${ }^{4), 5), 6), 13), 17)}$ equations for one-dimensional free-surface flow. The equations 
are the continuity equation or the mass conservation equation, i.e.,

$$
\frac{\partial y}{\partial t}+\frac{1}{b_{s}} \frac{\partial Q}{\partial x}=0
$$

and the momentum equation, i.e.,

$$
\frac{\partial Q}{\partial t}+\frac{\partial}{\partial x}\left(\frac{\alpha Q^{2}}{A}\right)+g A \frac{\partial y}{\partial x}+g A S_{f}=0
$$

where $t$ is time, $x$ is distance along the longitudinal axis of watercourse, $A$ is cross-sectional area, $y$ is water surface elevation, $\alpha$ is momentum correction coefficient, $b_{s}$ is storage width, $g$ is the gravitational acceleration and $S_{f}$ is friction slope. The $S_{f}$ value can be evaluated using Manning's formula:

$$
S_{f}=\frac{n^{2} Q|Q|}{A^{2} R^{4 / 3}}=\frac{Q|Q|}{K^{2}}
$$

in which $n$ is the Manning coefficient of frictional resistance, $R$ is hydraulic radius, $K$ is the conveyance factor.

For a river network, continuity of discharge and compatibility of energy levels must be satisfied at a junction $^{4), 13)}$;

$$
\begin{gathered}
\sum_{k=1}^{m} Q_{k}=0 \\
y_{1}+\frac{1}{2 g}\left(\frac{Q_{1}}{A_{1}}\right)^{2}=y_{2}+\frac{1}{2 g}\left(\frac{Q_{2}}{A_{2}}\right)^{2}=\ldots \\
=y_{k}+\frac{1}{2 g}\left(\frac{Q_{k}}{A_{k}}\right)^{2}=y_{m}+\frac{1}{2 g}\left(\frac{Q_{m}}{A_{m}}\right)^{2}
\end{gathered}
$$

where $m$ is the total number of links, which emanate from the junction, $Q_{k}$ is the discharge of the $k$-th link.

Hydraulic structures along or adjacent to the watercourse such as dams, weirs, bridges or waterfalls are considered as internal boundaries. Two equations equivalent to the St. Venant equations are required. They $\operatorname{are}^{13), 18)}$ :

$$
\begin{gathered}
Q_{j}^{i+1}-Q_{j+1}^{i+1}=0 \\
Q_{j+1}^{i+1}=\Phi\left(y_{j}^{i+1}, y_{j+1}^{i+1}\right)
\end{gathered}
$$

where $i$ denotes time line and $j$ denotes space line in the discrete solution domain. $j$ and $j+1$ denote two computational points located immediately upstream and downstream of the structure so that the change of storage at the structure can be ignored.

Equation (7) is usually an empirical relation for computing discharge values through the structures.

\section{SIMULTANEOUS SOLUTION}

\section{(1) Discretization and double-sweep method}

Equations (1) and (2) can be solved numerically by approximating them with a set of finite difference algebraic equations. In this paper, the widely-used Preissmann implicit finite difference scheme $^{5,6), 13), 17)}$ is applied.

The equations expressed in finite difference for a $\Delta x$ river reach from cross section $j$ to cross section $j+1$ can be written as follows ${ }^{5), 6), 13)}$ :

$$
\begin{aligned}
& A_{1} \Delta y_{j+1}+B_{1} \Delta Q_{j+1}+C_{1} \Delta y_{j}+D_{1} \Delta Q_{j}+G_{1}=0 \\
& A_{2} \Delta y_{j+1}+B_{2} \Delta Q_{j+1}+C_{2} \Delta y_{j}+D_{2} \Delta Q_{j}+G_{2}=0
\end{aligned}
$$

where $\Delta y$ and $\Delta Q$ are increments of water surface elevation and discharge during the time step $\Delta t$. $A_{1}$, $B_{1}, \ldots, G_{2}$ can be computed from the known values of $Q$ and $y$ at the previous time step.

A convergent discretized form of the basic relationships, together with appropriate boundary conditions, furnishes a system of non-linear algebraic equations in terms of unknown flow variables at the next time. The double-sweep method ${ }^{4), 55,6), 7), 13), 15), 16), 17)}$ is usually used because of its advantages.

By introducing the linearized approximation ${ }^{4), 5), 13)}$ :

$$
\Delta Q_{j}=E_{j} \Delta y_{j}+F_{j}
$$

which is inserted into the difference equations (8) and (9), yielding:

$$
\Delta y_{j}=L_{j} \Delta y_{j+1}+M_{j} \Delta Q_{j+1}+N_{j}
$$

As its name, the double-sweep method includes two "sweeps" along a river reach. The first sweep is forward sweep or elimination sweep and the second sweep is backward sweep or substitution sweep. In other words, there are two steps in calculation process. In the first step, the coefficients $E, F, L, M$, $N$ are calculated for all cross sections (computational points) of the reach and then the unknown values of $Q$ and $y$ are obtained by back substitution in the second step. The double-sweep method is now widely accepted as the most efficient way to solve system of nonlinear as well as linear equations ${ }^{17}$.

\section{(2) Determining the order for double-sweep}

The double-sweep method can be applied for dendritic or branched river networks ${ }^{7), 13), 15), 16)}$ (i.e. the network without loop) with some modifications. For branched networks, the main problem is determining the order for elimination in forward sweep. That is, the network will be rearranged so that the doublesweep method can be applied as in the case of a single reach. In this paper, a procedure used to solve this problem automatically is developed.

The river network is modeled by a basic element of river reach or branch (i.e., link in graph theory). Each reach has two nodes at two ends. The reach's index and node's index are independently numbered as integer numbers. There is no strict criterion for 
numbering river reaches and nodes, except the number of a reach (or a node) must not be coincided with others. A node is a position of junction (confluence) or external boundary. As double-sweep method is applied only to subcritical flows, it is not necessary to make distinction between upstream and downstream boundary in the elimination process. Thus, the "positive" flow direction in a reach from one node to the other is supposed firstly. When the computation completed, if the value of discharge is negative, it means that the real flow direction in the reach is opposite with the supposed one. By this way, the river network can be described by a set of river reaches and then the respective nodes. Mathematically, it can be described by two vectors or two lists. The first list is the list of river reach's indexes (ListR) and the second one is the list of node's indexes ( $L i s t N)$. The second depends upon the first and has a relationship with the first: if the ListR has $n$ elements, the $L i s t N$ will have $2 n$ elements.

The two lists are set up simply. At first, the ListR is initiated with free order. From the ListR and respective supposed flow direction in each reach, the $L i s t N$ is constructed. The procedure to look for the order of elimination now will work with these two lists. Its substance is the alternative progress of searching unique nodes in the List $N$ and removing the respective reach from ListR until List $N$ is empty. It is explained by the flowchart in Fig. 1. The orders of reaches that are eliminated successively, as well as the direction of elimination in each reach are both formed by this procedure. They are ListROrder and ListNOrder, respectively. Considering an example of river network as shown in Fig. 2 and using the procedure, following lists are yielded:

ListR (at beginning): $\{1,2,3,4,5,6,7,8,9,10,11$, $12,13,14,15,16,17,18,19,20,21,22,23,24,25$, $26,27\}$

ListN (at beginning): $\{1,3 ; 2,3 ; 3,8 ; 4,8 ; 5,7 ; 6$, $7 ; 7,8 ; 8,13 ; 9,11 ; 10,11 ; 11,13 ; 12,11 ; 17,12$; 18,$12 ; 15,14 ; 16,14 ; 14,13 ; 20,21 ; 13,21 ; 19$, $21 ; 22,21 ; 23,22 ; 24,22 ; 25,21 ; 26,28 ; 27,28 ; 28$, 21\}

and, after the procedure has done:

ListROrder: $\{1,2,4,5,6,9,10,13,14,15,16,18$, $20,22,23,24,25,26,3,7,8,12,17,21,27,19,11\}$ ListNOrder: $\{\mathbf{1}, \mathbf{3}, \mathbf{2}, \mathbf{3}, \mathbf{4}, \mathbf{8}, \mathbf{5}, \mathbf{7}, \mathbf{6}, \mathbf{7}, \mathbf{9}, \mathbf{1 1}, \mathbf{1 0}, \mathbf{1 1}$, $17,12,18,12,15,14,16,14,20,21,19,21,23,22$, $24,22,25,21,26,28,27,28,3,8,7,8,8,13,12,11$, $14,13,22,21,28,21,21,13,11,13\}$

For easy explanation, italic numbers are used for reaches and bold numbers are used for nodes in the lists. In addition, the commas and the semi-colons are alternatively used in the ListN (at beginning) with the purpose of easily recognizing the relationship
Initiate two lists: ListROrder, ListNOrder; ListROrder=Empty, ListNOrder=Empty.

Take ListR and ListN into consideration

Search in ListN for the unique nodes. With each unique node:

- put it into ListNOrder and then remove it from ListN

- find the correspond reach and put it into ListROrder and then remove it from ListR.

- find the other node of the reach and put it into ListNOrder and then remove it from ListN.

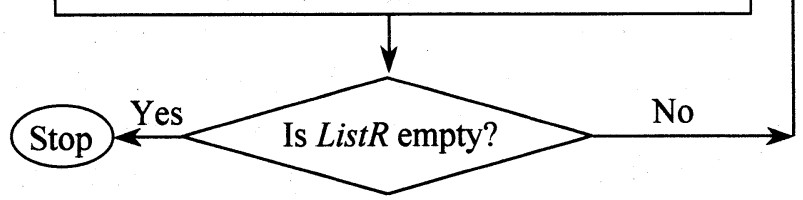

Fig. 1 Flow-chart of the procedure determining the order of elimination

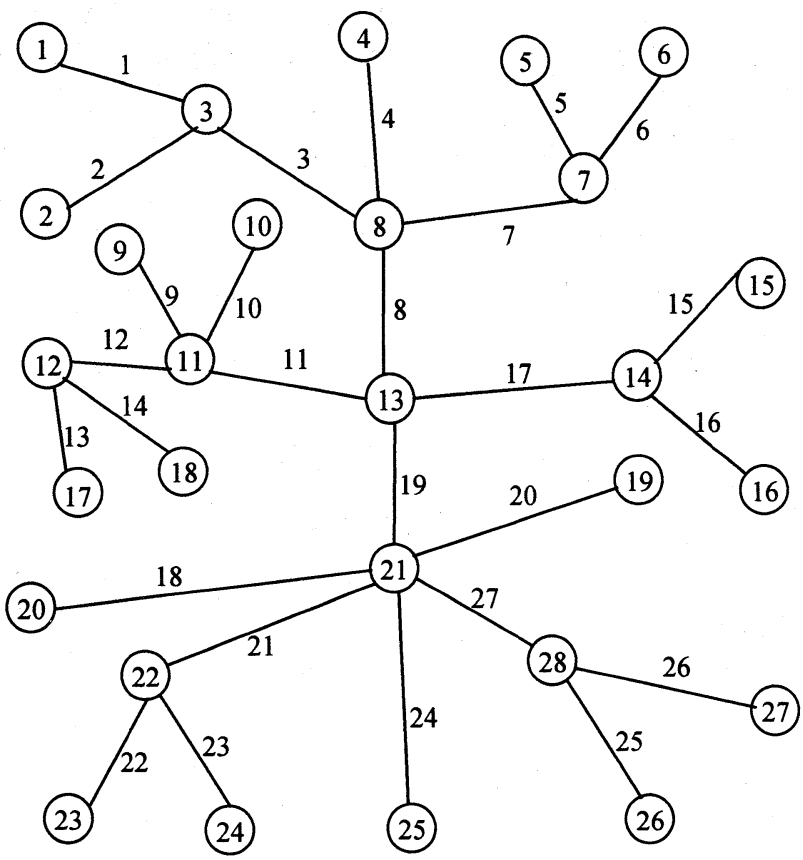

Fig. 2 An example of river network described by river reaches and nodes

between reaches and respective nodes, regardless the validation as regular form used in mathematics.

\section{(3) Modifying the lists}

Depend on the two lists (ListR and $L i s t N$ ) supplied as input information; the procedure always generates two respective lists automatically (ListROrder and ListNOrder). A problem can occur when the last appeared element in ListNOrder (last node) is not a boundary node. In this case, the solution at this last node (e.g. node 13 in preceding example) must be 
resolved theoretically by solving the equations (4) and (5) written for this node. This should lead a complexity in computation, so in this paper the two lists (ListROrder and ListNOrder) need to be modified and rearranged. Because of branched network, there is unique path between two nodes. The paths from the last node in ListNOrder to each of boundary nodes can be found out by using the standard "shortest-path algorithm" shortest path of these paths will be used to rearrange the order of ListROrder. The way to rearrange is that all river reaches in this shortest path will be moved to the end of the ListROrder and the ListNOrder will be respectively rearranged, but the direction is reversed to the supposed one. At last, the new ListROrder and new ListNOrder are received. Combining these two lists by mixing the elements with the form as node $\rightarrow$ river reach $\rightarrow$ node $\rightarrow$ node $\rightarrow$ river reach $\rightarrow \ldots \rightarrow$ node, the final order for computation is found out, named FinalList. Now, the elimination sweep is controlled by FinalList. For simplification, a downstream boundary condition is predefined and is supplied as an input data in this paper and the only one path has to look for is the path from last node in ListNOrder to this predefined node. For example, if the predefined node is 25 (Fig. 2) so the path is $\mathbf{1 3} \rightarrow 19 \rightarrow \mathbf{2 1} \rightarrow \mathbf{2 1} \rightarrow 24 \rightarrow \mathbf{2 5}$; and the rearranged ListROrder and ListNOrder are:

ListROrder: $\{1,2,4,5,6,9,10,13,14,15,16,18$, $20,22,23,25,26,3,7,8,12,17,21,27,11,19,24\}$

ListNOrder: $\{\mathbf{1}, \mathbf{3}, \mathbf{2}, \mathbf{3}, \mathbf{4}, \mathbf{8}, \mathbf{5}, \mathbf{7}, \mathbf{6}, \mathbf{7}, \mathbf{9}, \mathbf{1 1}, \mathbf{1 0}, \mathbf{1 1}$, $17,12,18,12,15,14,16,14,20,21,19,21,23,22$, $24,22,26,28,27,28,3,8,7,8,8,13,12,11,14,13$, $22,21,28,21,11,13,13,21,21,25\}$

and the FinalList is:

FinalList: $\{1,1,3,2,2,3,4,4,8,5,5,7,6,6,7,9$, $9,11,10,10,11,17,13,12,18,14,12,15,15,14$, $16,16,14,20,18,21,19,20,21,23,22,22,24,23$, $22,26,25,28,27,26,28,3,3,8,7,7,8,8,8,13$, $12,12,11,14,17,13,22,21,21,28,27,21,11,11$, 13, 13, 19, 21, 21, 24, 25$\}$

From the FinalList, the elimination sweep can be read as follows: start to calculate the coefficients at node 1 , and then, calculate the coefficients for all computational points along reach 1 to node 3 . After that, move to node 2 and calculate the coefficients at node 2 , and calculate the coefficients for reach 2 to node 3 , and so on. When elimination process is completed, the substitution process is done with revered order in FinalList. At last, the unknowns can be found out for all computation points of the network. The standard double-sweep for a single river reach requires two boundary conditions at two ends of the reach. In river networks, there are two kinds of reaches: reaches with one boundary condition at one end, and reaches without any boundary condition at both two ends. Therefore, all reaches need to be processed so that the coefficients $E, F$ can be computed at the first cross section in elimination sweep and $L, M, N$ at the last cross section in substitution sweep. This problem can be solved by using nodes close to these cross sections. That is the reason why the FinalList is constructed as above form. Using the procedure to create the order for double-sweep method, the internal nodes have been treated so that there are one or more incident reaches coming to a node but only one reach emanating from the node in forward sweep. Thus, the main rule for computation at an internal node is that all incident reaches of this node have been swept to find out the values of coefficients $E$ and $F$ at the last cross section of these incident reaches. From these values, the coefficient $E$ and $F$ can be computed for the reach emanating from the node $^{13)}$ and they are the values of first cross section in next reach which will be eliminated.

The preceding treatment for a river network is essentially considering it as a river reach, but a special river reach with special cross sections. These special cross sections have additional computations of nodes. In other words, when the last order has been set up, all the reaches in the networks have been rearranged to make the only one new reach. Physical connections at a junction are transferred to treatment of nodes and their close cross sections in simulation. Therefore, from this main idea, it can be said that all of the effectiveness of double-sweep method for a single river reach can be inherited in the case of river networks. With the improvement, the double-sweep method can be applied for any branched networks regardless the maximum order of a branch as well as the number of branches at a node. When a river network is changed, it is not necessary to do any modification of basic formations or source codes. All simple works have to do are re-describing the network by branches and respective nodes and supply them to a model as input information.

\section{APPLICATION AND RESULTS}

The model is applied for a part of the Red river basin, a significant basin in Vietnam. The map of this part is shown in Fig. 3. The investigated river network is described by reaches and nodes as shown in Fig. 4. The total length of the river reaches using in computation is about $900 \mathrm{~km}$ with 262 cross sections. The distance between a cross section to adjoining one varies from $400-500 \mathrm{~m}$ to $3-4 \mathrm{~km}$, even to $6 \mathrm{~km}$. The typical shape of cross sections in the network is the form of compound section (Fig. 5). The network is virtually dyked with extensive floodplains about 200- 


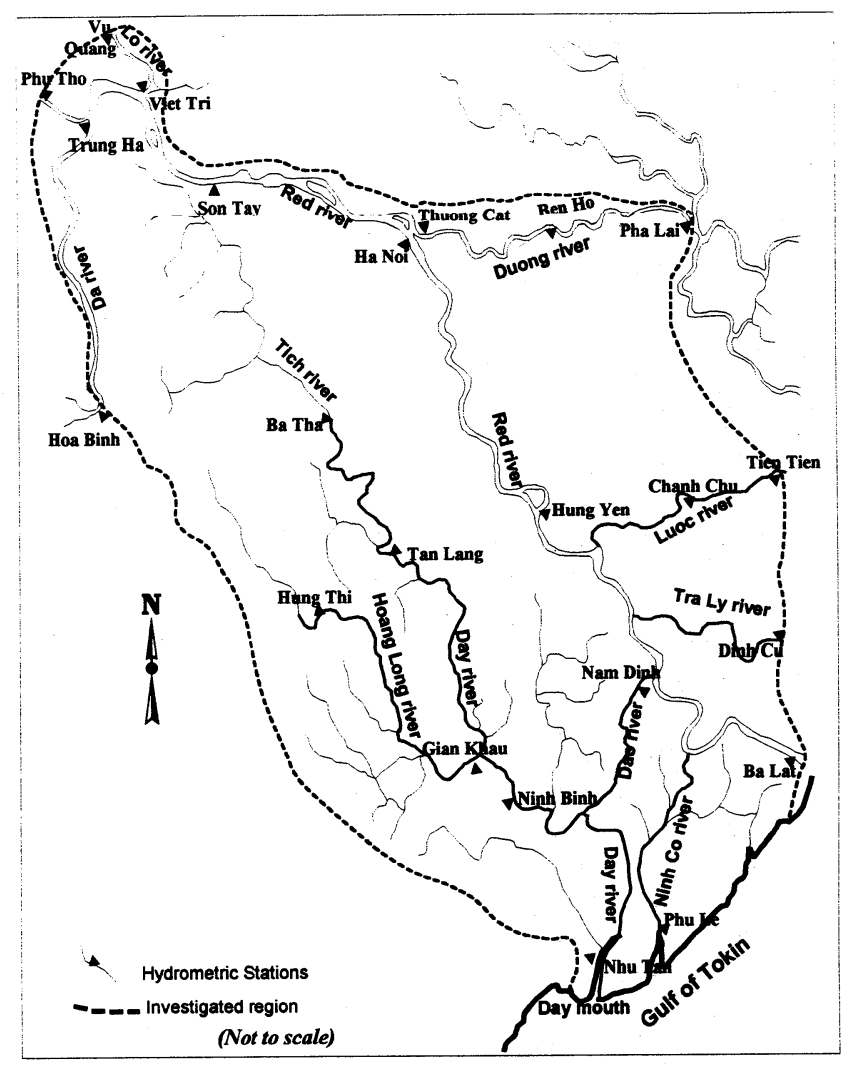

Fig. 3 Map of the investigated region

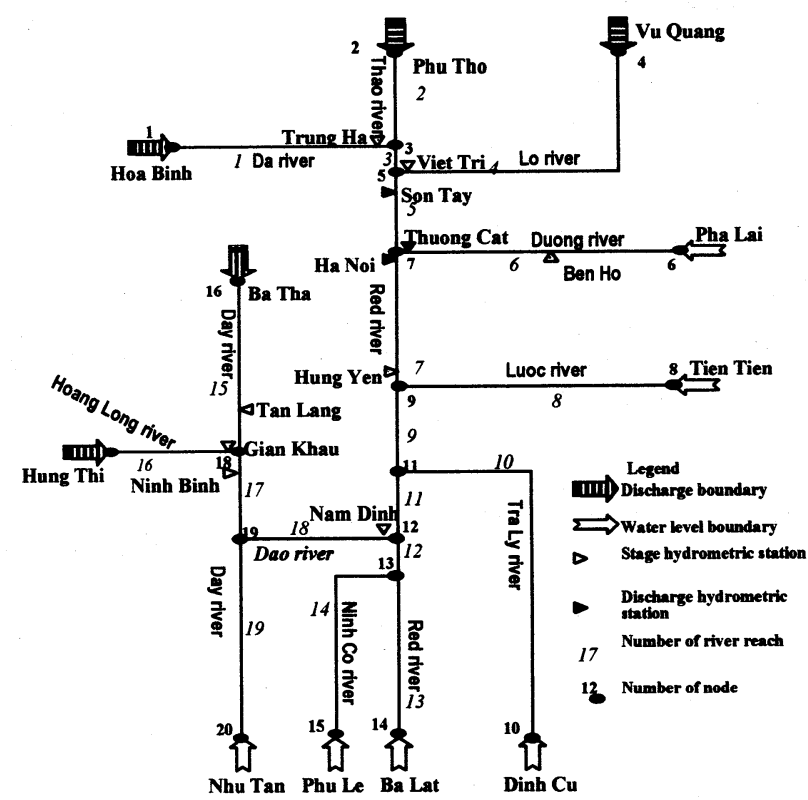

Fig. 4 Schematic of investigated river network

$300 \mathrm{~m}$; especially $2-3 \mathrm{~km}$ at some places. The floodplain is cultivated area. The cover conditions of floodplain vary strongly in space and time. The flow regime in the region is affected by both river flow and tide from the sea and the bottom slope of riverbed is mild (about $0.5-0.01 \%$ ). For these cross sectional properties, the conveyance factor $(K)$ can be evaluated partly, including left floodplain, main channel and right floodplain. The total cross-sectional conveyance factor is calculated as follows ${ }^{6}$ :

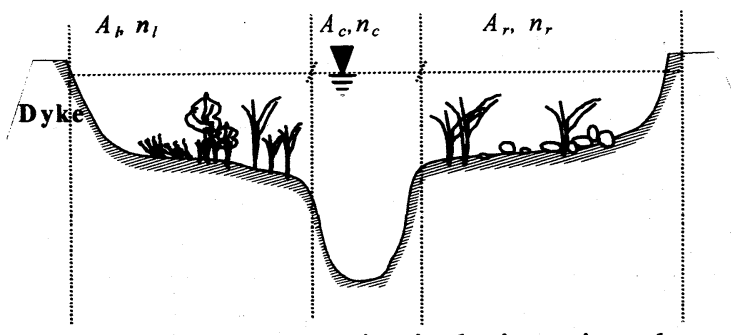

Fig. 5. Typical cross section in the investigated network
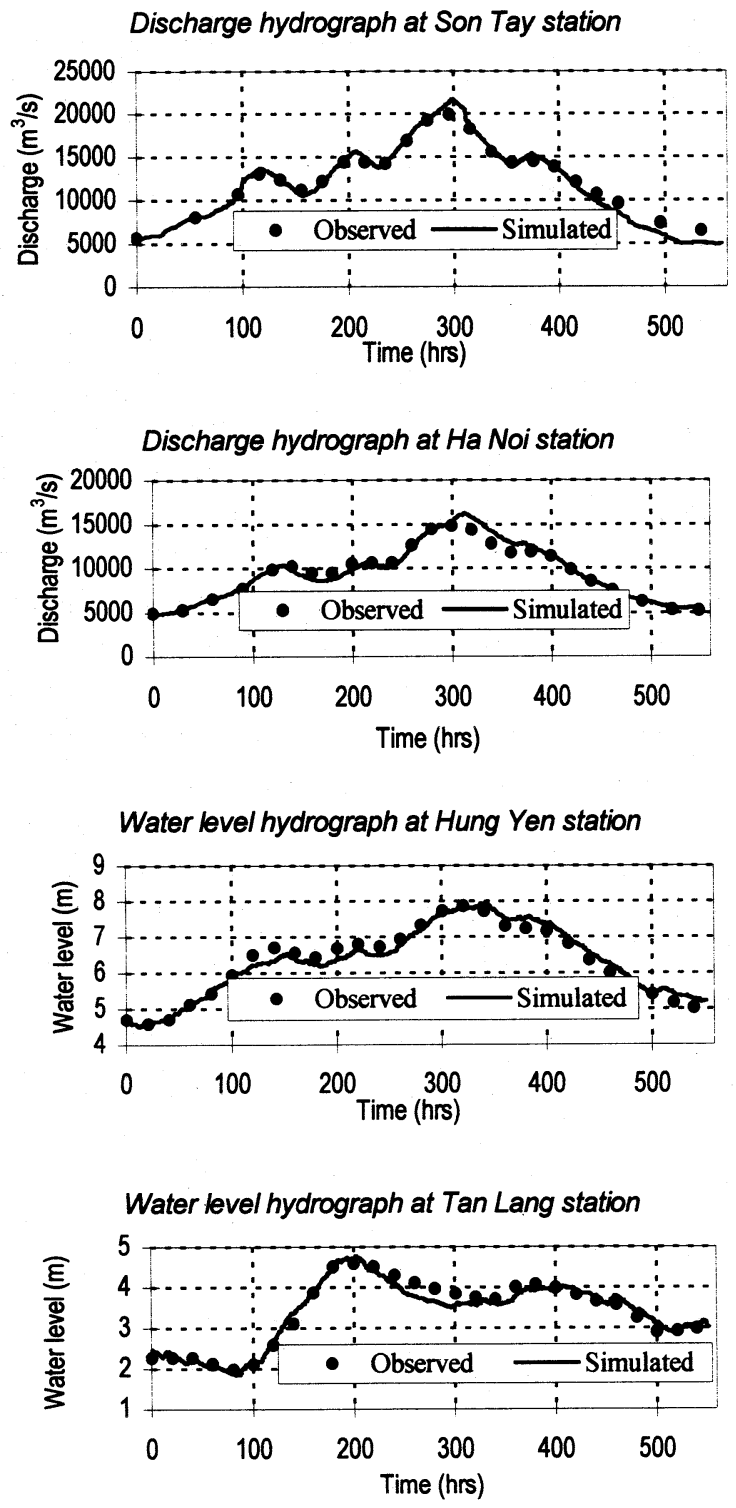

Fig. 6 Results from the model applied for a part of the Red river network.

$$
K=K_{l}+K_{c}+K_{r}
$$

in which the subscripts $l, c$ and $r$ designate left floodplain, main channel and right floodplain.

After Finallist for the network is created, the double-sweep is used incorporate with boundary conditions. The solution for discharge and water level can be found out from equations (10) and (11) for all computational points in the network. The flood from 9 to 31 , August 1996 is taken into calibration process. 
Table 1 The values of Manning's $n$ for some types of floodplain cover condition

\begin{tabular}{l|c|c}
\hline $\begin{array}{c}\text { Type of floodplain } \\
\text { cover condition }\end{array}$ & $\begin{array}{c}\text { The initial } \\
\text { values of } n\end{array}$ & $\begin{array}{c}\text { The selected } \\
\text { values of } n\end{array}$ \\
\hline $\begin{array}{l}\text { Villages or } \\
\text { residential areas }\end{array}$ & 0.080 & $0.070-0.160$ \\
\hline Bamboo hedges & 0.050 & $0.045-0.100$ \\
\hline $\begin{array}{l}\text { Grass or cultivated } \\
\text { area with cereals }\end{array}$ & 0.035 & $0.020-0.050$ \\
\hline $\begin{array}{l}\text { Fluvial bogs with } \\
\text { sedges or jutes }\end{array}$ & 0.030 & $0.025-0.050$ \\
\hline Main channel & 0.020 & $0.015-0.035$ \\
\hline
\end{tabular}

This flood was a large flood in the Red river basin. Upstream boundary conditions used in calibration are given as observed discharge hydrographs at Hoa Binh, Phu Tho, Vu Quang, Hung Thi and $\mathrm{Ba}$ Tha. The downstream boundary conditions are given as observed water level hydrographs at Nhu Tan, Phu Le, Ba Lat, Dinh Cu, Tien Tien and Pha Lai (Fig. 4). The investigated and measured topographical data from 1992-1999 are used in simulation.

As the dynamic unsteady flow model is often sensitive to the value of the Manning coefficient of frictional resistance $(n)$, the calibration process is adjusting value of $n$ to produce the minimum differences between observed and simulated hydrographs of discharge and water level. The values of $n$ are selected via trial-and-error calibration methodology. Initial values and adjustments of $n$ are based on field investigations and other guidelines ${ }^{18), 20}$. The initial and selected values of $\boldsymbol{n}$ for typical types of floodplain cover condition as well as for main channel part in the network are shown in Table 1.

Results of calibration are shown in Fig. 6. The error in discharge is $4-15 \%$. The maximum error in peak water level is $42 \mathrm{~cm}$ and the maximum error in time of the peak is 10 hours. From these results, it can be said that the model with the improvement of automatically determining the order for doublesweep can more conveniently use in flood routing in branched river network.

\section{CONCLUSIONS}

An improvement of simultaneous solution for dynamic flood routing in branched network by Preissmann implicit finite difference scheme is introduced in this paper. With the consideration that a river reach is the basic element for river network modeling, a procedure of determining the order for double-sweep algorithm is developed. By this procedure, the model can be applied to complex branched networks regardless the number of reaches at a node. This permits to "complicate" river network without any modification of the model, excepting input data. The model developed from the improvement is constructed and applied for routing a flood in a part of the Red river basin. The calculated values have good agreements with observed ones.

However, it is necessary to apply the model to other river networks in order to estimate its applicability more thoroughly. The improvement presented here is un-applicable for a completed looped network and this is still a disadvantage of the simultaneous solution based on double-sweep method.

\section{REFERENCES}

1) Singh, V.P.: Hydrologic systems, Vol. 1 Rainfall-Runoff Modeling, Prentice Hall, 1988

2) Hydrologic Engineering Center: Hydrologic Modeling System (HEC-HMS) Technical Reference Manual, US Army Corps of Engineers, 2000.

3) Joliffe, I.B.: Computation of dynamic waves in channel network, J. of Hydraulic Engineering, pp. 1358-1370, Vol. $110(10), 1984$.

4) Lai, C.: Numerical modelling of unsteady open-channel flow in Advances in Hydroscience (Edited by Yen B. C.), pp. 161-333, Vol. 14, 1986.

5) Mahmood, K. and Yevjevich, V.: Unsteady in openchannels, Vol. 1, Water Resour. Publ., Fort Collins, 1975

6) Segio Montes: Hydraulics of open-channel flow, American Society of Civil Engineers (ASCE) Press, USA, 1998.

7) Choi, G. W. and Molinas, A.: Simultaneous solution algorithm for channel network modeling, Water Resour. Res., pp. 321-328, Vol. 29(2), 1983.

8) Fread, D.L.: Technique for implicit dynamic routing in river with tributaries, Water Resous. Res., pp. 918-926, Vol. $9(4), 1973$.

9) Ankan, A.O. and Yen, B.C.: Diffusion wave flood routing in channel networks, J. of Hydr. Div., ASCE, pp. 719-732, Vol. 107 (7), 1981.

10) Inoue, K., Toda, K. and Maeda, O.: Inundation model in the region of river network system and its application to Mekong delta, Annual Journal of Hydraulic Engineering, JSCE, pp. 485-490, Vol. 44, 2000 (in Japanese).

11) Booij, N.: Report on the ICES subsystem FLOWS, Report No. 78-3, Delft University of Technology, 1980.

12) Watanabe, K.: Basic study and application on water control in low-land open-channel networks with various hydraulic structures, Research report of Grant-in-aid scientific research (C), No. 07650605, 1997 (in Japanese)

13) Cunge, J.A., F.M. Holly, Jr. and Verway, A.: Practical aspects of computational river hydraulics, Pitman, 1980.

14) Kanemoto, M. and Tsunematsu, Y.: Graph-theoretic analysis of gradually varies unsteady flows in river channel networks, J. of Hydra., Coastal and Envir. Engineering, JSCE, pp. 67-75, No. 429/II-15, 1991 (in Japanese).

15) Ping, P. and Xiaofang, R.: Method of flood routing in multibranch rivers, $J$. of Hydraulic Engineering, ASCE, pp. 271-276, Vol. 125(3), 1999.

16) Nguyen, Q.K. and Kawano, H.: Simultaneous solution for flood routing in channel networks, $J$. of Hydraulic Engineering, ASCE, pp. 744-750, Vol. 121(10), 1995.

17) Abbott, M.B. and Basco, D.R.: Computational fluid dynamics - an introduction for engineers, Longman Scientific \& Technical and John Wiley \& Sons, 1989.

18) Fread, D.L.: NWS FLDWAV model: Theoretical description, National Weather Service, USA, 1998

19) Baase S.: Computer Algorithms - Introduction to Design and Analysis, Addison-Wesley Publ. Company, 1988.

20) Yen, B.C., ed.: Channel flow resistance - Centennial of Manning's formula, Water Res. Publications, USA, 1992.

(Received October 2, 2000) 